\title{
SOBRE ZUMBIS E SUJEITOS DOTADOS DE MENTES: O CETICISMO DE DRETSKE ACERCA DO CONHECIMENTO DE SI *
}

On zombis and subjects gifted with minds: Dretske's skepticism on self-knowledge

Karla Chediak **
Edgar Marques ***

Resumo: No presente artigo, apresentamos nossa interpretação dos argumentos fornecidos por Fred Dretske para fundamentar seu ceticismo acerca da possibilidade de conhecermos introspectivamente que possuímos estados mentais e que, portanto, não somos zumbis. Mostramos que a posição cética acerca do conhecimento de si adotada por Dretske tem como fundamento a sua concepção externalista e informacional de todos os estados mentais e que essa forma de conhecimento demanda um outro tipo de abordagem.

Palavras-chave: Dretske. Introspecção. Autoconsciência. Autoconhecimento. Zumbis.

Abstract: In this article, we present our interpretation of the reasons given by Fred Dretske to support his skepticism about the possibility of knowing introspectively that we have mental states and that therefore we are not zombies. We show that Dretske's skeptical position on self-knowledge is based on his

\footnotetext{
* Gostaríamos de agradecer à parecerista anônima ou ao parecerista anônimo, cujas valiosas sugestões possibilitaram corrigir algumas das deficiências presentes na versão inicial deste texto. Artigo recebido em 19/12/2016 e aprovado para publicação em 15/06/2017.

** Professor Associado da Universidade do Estado do Rio de Janeiro; doutor em Filosofia pela Universität Konstanz (1995); pesquisador do CNPq.

*** Professora Associada da Universidade do Estado do Rio de Janeiro (UERJ); doutora em Filosofia pela Pontifícia Universidade Católica do Rio de Janeiro (1995). pesquisador do CNPq.
} 
externalist and informational conception of all mental states and that this form of knowledge demands another approach.

Key-words: Dretske. Introspection. Self-consciousness. Self-knowledge. Zumbis

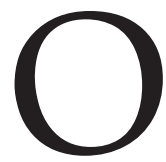

senso comum e o bom senso filosófico compartilham de uma compreensão da realidade segundo a qual cada um de nós possui um conhecimento de si que abrange o conjunto dos próprios estados mentais ocorrentes de natureza representacional. Isso significa simplesmente que, segundo essa concepção, cada um de nós sabe o que pensa e também que sabe que pensa isso que pensa. Uma das características principais desse conhecimento de si consiste em sua independência da realização de quaisquer investigações empíricas dos próprios estados psicológicos internos ou dos próprios comportamentos para se constituir, envolvendo, pelo contrário, única e exclusivamente um acesso direto, imediato e privilegiado a esses dois objetos de conhecimento, a saber, aos conteúdos dos próprios pensamentos, por um lado, e ao fato de que esses pensamentos são por nós pensados, por outro. Assim, de acordo com essa compreensão, sabemos por via introspectiva o que pensamos e também sabemos que somos seres dotados de pensamentos, pois temos consciência de que pensamos os pensamentos que pensamos.

Pode ser, se os mais terríveis cenários céticos no que respeita às outras mentes venham a se mostrar verdadeiros, que vivamos em um mundo habitado por zumbis, isto é - de acordo com o uso dessa expressão na filosofia contemporânea de matriz anglo-saxã - por seres que se comportam como se fossem dotados de uma vida mental interna, mas que, na verdade, são desprovidos de uma mente ${ }^{1}$. Na medida em que não temos acesso aos estados mentais internos dos outros indivíduos, mas sim apenas ao seu comportamento aparente, parece ser legítima a pergunta pelas garantias que podemos ter de que os outros seres humanos de fato possuem uma vida mental interna, e não simplesmente se comportam, em função de causas que se nos escapam completamente, como se a tivessem, apesar de efetivamente não a ter.

Talvez os outros sejam zumbis, mas sabemos, ou cremos saber, de forma direta e imediata - vale dizer, por introspecção - que pelo menos nós não o somos. Essa parece ser uma das certezas basilares constitutiva da visão de mundo compartilhada pela maioria de nós, filósofos ou não filósofos. É precisamente essa certeza de que sabemos por meio de um acesso introspectivo que nós não somos zumbis, isto é, de que sabemos direta

\footnotetext{
${ }^{1}$ Adotamos neste artigo a concepção dretskeana de mente, tal como ele expõe no prólogo de seu livro Naturalizing the mind, quando diz que todos os fatos mentais são fatos representacionais e todos os fatos representacionais são fatos sobre funções informacionais.
} 
e imediatamente que pensamos pensamentos dotados de determinados conteúdos representacionais ${ }^{2}$, que Fred Dretske, em uma série de artigos publicados entre 1999 e $2012^{3}$, surpreendentemente coloca sob suspeição. Ele defende uma posição cética acerca da possibilidade de que possa haver um conhecimento de si introspectivo e que possamos, portanto, saber de forma imediata e direta que não somos zumbis. . No presente artigo, apresentaremos nossa interpretação das razões fornecidas por Fred Dretske para fundamentar esse seu, inusual, ceticismo acerca do conhecimento próprio a cada um de nós de que possuímos uma mente, de que não somos zumbis.

A formulação da dúvida cética de natureza epistêmica acerca da existência de outras mentes além da nossa própria é estruturalmente semelhante à formulação da dúvida cética acerca da existência do mundo exterior. $\mathrm{O}$ ponto de partida da dúvida relativa à existência de um mundo objetivo fora de nós consiste na adoção de uma postura de desconfiança em relação às informações acerca desse mundo oferecidas a nós pelas sensações e percepções. O argumento cartesiano do sonho, formulado na primeira das Meditações Metafísicas, e que aponta para a inexistência de marcas internas às percepções que permitam diferenciá-las, no momento da sua ocorrência em nós, das representações oníricas ou dos delírios, fornece, de maneira paradigmática, razões para colocarmos em dúvida a existência do mundo físico, de tal modo que inúmeros filósofos posteriores a Descartes, como Kant, por exemplo, tentaram responder ao desafio colocado por ele, formulando argumentos que provariam cabalmente a existência do mundo exterior.

O procedimento adotado na formulação dessas duas dúvidas céticas clássicas é, assim, praticamente o mesmo: um certo tipo de saber é caracterizado como mais imediato e direto por contraposição a um outro tipo de saber, que se considera ser obtido de uma forma mais indireta e mediada. Depois argumenta-se que esse pretenso conhecimento que envolve mediação e que não se realiza de forma direta somente pode ser completamente justificado caso ele se fundamente naquele outro tipo de conhecimento, mais direto e

\footnotetext{
${ }^{2}$ Utilizamos as expressões "conteúdos representacionais" e "conteúdos informacionais" de forma indiscriminada. No entanto, cabe assinalar que Dretske reconhece que há dois tipos de informação: analógica e digital. Apenas a informação digital é cognitiva, por ser conceitual. Nesse artigo, referimo-nos sempre à informação digital, aquela que pode ser expressa numa proposição.

${ }^{3}$ Ver bibliografia.

${ }^{4}$ Como veremos com maior clareza no desenvolvimento do presente artigo, o ceticismo de Dretske não abarca a dúvida acerca de sermos entes dotados tanto de vida mental efetiva quanto do conhecimento desse fato. Ele em nenhum momento duvida que nós não somos zumbis e que nós sabemos que não o somos. O objeto da dúvida é, assim, não o conhecimento que temos acerca de nós mesmos, mas sim, mais propriamente, o modo como chegamos a obter esse conhecimento, isto é, a dúvida de Dretske diz respeito unicamente à fonte do conhecimento de si (self-knowledge). É por essa razão que Dretske caracteriza sua própria posição como sendo a de um ceticismo conciliatório (DRETSKE 2012a, p. 50), em contraposição a um ceticismo acerca da existência do conhecimento de si.
} 
mais imediato. O passo seguinte consiste em mostrar que o conhecimento mais direto e mais imediato, ao contrário do que julga o senso comum, não fornece apoio suficiente para que se sustente o conhecimento indireto e mediato, o que faz com que este passe a ser sujeito a dúvidas ${ }^{5}$. Dessa forma, como indivíduos dotados de sensibilidade que somos, temos impressões sensíveis às quais acedemos direta e imediatamente, mas que por si só não podem garantir que existam os objetos que elas apresentam para nós como possuindo tal ou tal qualidade. Isso parece poder conduzir a um estado de dúvida acerca de uma existência real do mundo constituído por tais objetos. Da mesma maneira, os estados mentais dos outros sujeitos parecem apresentar-se a nós não de forma direta, mas sim unicamente através da observação dos comportamentos físicos e verbais assumidos por esses sujeitos. Nós nos damos conta que eles se comportam de forma semelhantes a nós quando nos encontramos em determinados estados internos e inferimos que eles possuem estados internos semelhantes aos que identificamos em nós mesmos. O problema é que os comportamentos observados não parecem fornecer uma base suficientemente sólida para que possamos atribuir sem qualquer sombra de dúvida uma vida mental a esses sujeitos, dado que podemos conceber - isto é, não é logicamente contraditório - que esses comportamentos, no caso das outras pessoas, se realizem sem o acompanhamento desses estados internos.

A formulação dessas duas dúvidas tem como pressuposto, assim, uma oposição entre estados internos aos quais o sujeito acede imediatamente e estados de coisas cuja existência é inferida a partir dos primeiros, recaindo, então, a dúvida inelutavelmente sobre esses segundos. O cético parece ser nesse caso aquele que considera não ter razões que justifiquem a existência de um mundo exterior ou de outras mentes, mas que sabe acerca de si mesmo que ele possui sensações, percepções, pensamentos e demais estados mentais, sendo a partir do recurso a esses estados internos a ele que essas dúvidas são formuladas.

Gostaríamos, seguindo aqui uma longa e difundida tradição, de tomar Descartes como um caso paradigmático dessa concepção, apesar de não termos de maneira nenhuma a pretensão de abordar de forma mais rigorosa e aprofundada a concepção cartesiana, uma vez que Descartes desempenha no presente texto unicamente o papel de fornecer os traços gerais do ponto de vista criticado por Dretske.

Na primeira definição da exposição sintética de sua metafísica apensada às suas Segundas Respostas, Descartes caracteriza pensamento como sendo tudo aquilo que se encontra em nós mesmos de uma tal maneira que somos dele imediatamente conscientes, abarcando, assim, com esse

\footnotetext{
${ }^{5}$ Utilizamos o termo "sujeito" neste artigo para significar indivíduo capaz de pensamento proposicional sem nos comprometermos, contudo, com as implicações metafísicas associadas a esse termo.
} 
conceito o conjunto das representações do entendimento, da vontade, da imaginação e da sensibilidade 6 . Uma caracterização semelhante se encontra também no parágrafo 9 do primeiro livro dos Princípios de Filosofia, onde Descartes afirma o seguinte: "pelo termo pensamento compreendo tudo aquilo de que somos conscientes como sendo em nós na medida em que dele somos conscientes" ${ }^{\prime 7}$.

O ponto fundamental no conceito de "pensamento" assim fixado consiste, a nosso ver, na ideia de que a realização de um ato de pensamento implica a consciência desse próprio ato. $\mathrm{O}$ simples ato de pensarmos possibilitaria, então, que conhecêssemos imediata e diretamente o conteúdo desse ato e ainda o fato de que se trata de um conteúdo por nós pensado. Ao pensarmos o pensamento $P$ teríamos, dessa maneira, simultaneamente a consciência do conteúdo representacional $P$ e a consciência de que pensamos neste momento um pensamento com o conteúdo representacional $P$. Assim, quando pensamos que chove agora em Berlim sabemos, sem precisar realizar nenhum tipo de investigação empírica, qual é o conteúdo do nosso pensamento - isto é, que chove agora em Berlim - e que estamos pensando que chove agora em Berlim. Isso significa que, de acordo com essa concepção, ao nos encontrarmos em um determinado estado mental temos consciência imediata de seu conteúdo e também consciência imediata de que esse estado mental com esse conteúdo é precisamente o estado mental no qual nós nos encontramos neste momento. Ambos os conhecimentos - a saber, que pensamos em $\mathrm{P}$ e que pensamos que pensamos em $\mathrm{P}$ - são obtidos por introspecção e constituem, quando tomados em conjunto, o que normalmente consideramos ser autoconsciência ou conhecimento de si. Esse conhecimento é realizado em primeira pessoa e possui natureza autoritativa, pois não parece fazer sentido considerar que os sujeitos que se encontram nesses estados possam ser corrigidos por outros sujeitos no que diz respeito tanto ao conteúdo de seus pensamentos quanto ao fato de que eles estão pensando esses pensamentos. O sujeito que se encontra em um determinado estado mental desfruta, então, segundo essa visão, de uma autoridade especial no que diz respeito ao conhecimento/ consciência tanto do conteúdo desse estado quanto do fato de se encontrar em um estado mental com esse conteúdo.

\footnotetext{
6 "Thought. I use this term to include everything that is within us in such a way that we are immediately aware of it. Thus all the operations of the will, the intellect, the imagination and the senses are thoughts.", em: DESCARTES, R. (1985), The Philosophical Writings of Descartes, Vol. II, Cambridge University Press, Cambridge, pág 160. Texto em latim: “Cogitationis nomine complector illud omne quod sic in nobis est, ut ejus immediate conscii simus. Ita omnes voluntatis, intellectus, imaginationis \& sensuum operationes sunt cogitationes.", Descartes, R, Oeuvres de Descartes, Ed. C. Adam and P. Tannery,vol. VIII, Libraire Philosophique J. Vrin, Paris, 1964, p. 160. (As próximas referências à edição de Adam \& Tannery serão feitas usando a sigla AT seguida de algarismos romanos indicando o número do volume e de algarismos arábicos indicando os números das páginas).

7 "By the term "thought", I understand everything which we are aware of as happening within us, in so far as we have awareness of it." (DESCARTES 1985, vol. 2, p. 195). Texto em latim: "Cogitationis nomine, intelligo illa omnia, quae nobis consciis in nobis sunt, quatenus eorum in nobis conscientia est.", AT, VIII, 7.
} 
Fred Dretske coloca em questão justamente essa ideia cartesiana, aceita de forma tácita pela maioria de nós, de que conhecemos da mesma maneira - a saber, por meio de introspecção - o conteúdo de nossos pensamentos e crenças, por um lado, e o fato de que entretemos pensamentos e crenças com esses conteúdos, de outro. Vamos tentar primeiramente compreender o alcance da distinção sublinhada por Dretske entre esses dois tipos de conhecimentos para então avaliarmos as razões que ele aduz para fundamentar sua afirmação de que esses conhecimentos têm de ser obtidos por vias distintas.

Parece ser indisputável que cada um de nós tem acesso direto e imediato ao conteúdo de seus próprios pensamentos e crenças ocorrentes. Atribuir a esse acesso uma natureza direta e imediata significa apenas dizer que não precisamos, ao contrário do que se passa quando atribuímos pensamentos e crenças a terceiros, investigar empiricamente nossas ações e comportamentos para deduzirmos quais são as crenças e pensamentos que explicariam sua realização. Nós sabemos direta e imediatamente em que estamos pensando em um dado instante, podendo responder a uma pergunta a esse respeito sem precisarmos proceder a nenhum tipo de investigação empírica. Se Maria neste momento está pensando, por exemplo, que chove lá fora, então o conteúdo informacional próprio a esse pensamento está disponível imediatamente a ela e pode ser utilizado para guiar suas escolhas - ficar em casa ou então pegar um guarda-chuva ao sair - . De alguma maneira o conteúdo desse pensamento é acessado por Maria e acaba por contribuir para sua decisão de ficar em casa ou de pegar um guarda-chuva. O importante é que esse conteúdo proposicional tem de ser compreendido como disponibilizado para Maria prévia e independentemente do seu comportamento para que suas ações se deixem explicar através da atribuição a ela do pensamento - ou da crença - de que está chovendo. Quer dizer, se Maria pegou o guarda-chuva ao sair porque pensou que estava chovendo, então ela tem de ter tido algum acesso ao conteúdo desse pensamento de forma a agir levando em conta a informação que ele veicula, senão não faz sentido dizer que Maria pegou o guarda-chuva em função de sua crença de que estava chovendo. Isso não significa, obviamente, que esse acesso tenha de envolver necessariamente algum tipo de consciência reflexiva por parte do sujeito que se encontra nesse estado mental. Isto é, Maria não precisa ter consciência explícita de que ela entretém um pensamento com um determinado conteúdo para que ela efetivamente tenha esse pensamento. $O$ ponto central para a determinação de se o sujeito se encontra ou não em um determinado estado mental consiste simplesmente na consideração de se esse sujeito absorve ou não o conteúdo informacional veiculado por esse estado mental. O sujeito não precisa saber reflexivamente que absorveu um determinado conteúdo informacional para que essa absorção tenha ocorrido.

O que gostaríamos de enfatizar neste contexto é que atribuir a um sujeito acesso direto e imediato ao conteúdo dos seus estados mentais não significa considerar que esse sujeito necessariamente saiba de forma reflexiva que ele 
se encontra nesses estados mentais específicos, quer dizer, que ele esteja em condições de atribuir a si mesmo esses estados. Dizer que esse acesso é direto e imediato significa simplesmente avaliar que o sujeito tem disponível a si os conteúdos informacionais veiculados por esses estados sem que ele precise para isso realizar nenhum tipo de investigação empírica. Trata-se, em outras palavras, de uma caracterização meramente negativa dessa forma de acesso a esses conteúdos, que não nos obriga, para nossa sorte, a minudenciar o modo como esse acesso efetivamente se dá. Podemos, então, não sem certo alívio, abrir mão do apelo às metáforas visuais, abandonando o hábito de caracterizar a introspecção como uma espécie de visão interna daquilo que se apresentaria no palco da nossa interioridade.

O conteúdo de um pensamento ou crença que um sujeito tem está disponível a esse sujeito de uma maneira semelhante àquela pela qual está disponível a ele o conteúdo de suas percepções ${ }^{8}$, vale dizer, como algo que se dá a ele imediata e diretamente, sem que seja necessário que ele recolha informações de nenhum tipo acerca do mundo circundante para que ele tenha acesso ao conteúdo informacional veiculado por esse estado mental de natureza perceptual. Assim, por exemplo, quando Maria vê que a porta da casa está fechada, ela torna-se consciente por meio da percepção que um certo estado de coisas - uma determinada porta estar fechada - está presente no mundo, comportando-se, se ela for suficientemente arguta, de forma adequada a essa informação recebida. Maria, em função dessa informação visual, procura, digamos, a chave na sua bolsa e abre a porta, em vez de tentar atravessar a porta estando esta fechada. O ponto importante aqui é que Maria sabe que a porta está fechada meramente ao ver a porta, tornando-se, então, essa informação acerca do mundo disponível a ela na medida mesma em que essa representação visual é nela processada. Maria não deduz que vê a porta estar fechada nem descobre por meio de alguma investigação a posteriori que ela está vendo a porta fechada; ela pura e simplesmente vê a porta fechada, processando, então, essa informação obtida por meio da percepção, sem que nenhum outro tipo de operação mental que se desdobre sobre esse conteúdo informacional seja requerido.

Maria sabe, assim, que a porta está fechada simplesmente ao ver que a porta está fechada, sendo, dessa maneira, unicamente por meio da impressão visual que esse seu conhecimento se constitui. Esse conhecimento não deve ser confundido, contudo, com o conhecimento que Maria pode vir a ter de que ela está vendo a porta fechada. Uma coisa é saber através da visão que a porta está fechada, outra coisa bem diferente é saber que se está vendo que a porta está fechada. No primeiro caso, a informação veiculada refere-se à coisa vista por Maria, enquanto que a segunda informação é não apenas acerca do objeto visto, mas sim, em última instância, acerca da própria Maria, pois o conteúdo veiculado pelo segundo tipo de

${ }^{8}$ Dretske lança mão dessa comparação em inúmeros textos, como, por exemplo em Dretske (2003b) e Dretske (2004). 
conhecimento comunica a Maria que ela está tendo um determinado estado interno, a saber uma percepção visual com um determinado conteúdo. Maria nesse caso não apenas vê algo, mas, além disso, sabe que está vendo esse algo. A questão é se o modo pelo qual ela se informa visualmente acerca do mundo coincide com o modo pelo qual ela se informa acerca do estado interno em que ela se encontra.

O que o recurso a essas considerações acerca da natureza da percepção visual deve tornar claro nesse contexto é que a consciência dos conteúdos dos nossos estados mentais deve ser distinguida da consciência de que temos estados mentais com esses conteúdos. Assim, quando temos uma experiência perceptual verídica somos imediatamente conscientes dos objetos percebidos, o que significa dizer que as propriedades das quais nos tornamos conscientes através da percepção são propriedades das coisas percebidas, e não propriedades nossas. Para Dretske, os estados perceptuais que ocorrem nos diferentes sujeitos caracterizam-se exatamente por serem transparentes, isto é, por apresentarem características não do sujeito que os entretém, mas sim unicamente dos objetos intencionais acerca dos quais eles são. Nossas experiências perceptuais carregam informações acerca daquilo de que nos tornamos conscientes por meio delas, quer dizer, carregam informações acerca dos objetos que percebemos, mas elas não carregam em seu conteúdo a informação de que somos delas conscientes.

Tomando a percepção como paradigma da constituição e natureza dos estados internos representacionais em geral, incluindo aí, portanto, pensamentos e crenças, Dretske tenta, no conjunto de textos acima referido, exatamente argumentar em favor da tese de que devemos diferenciar a consciência que temos do conteúdo dos nossos estados mentais da consciência de que temos estados mentais com esses conteúdos.

Uma forma de destacar essa diferença envolve recordar que pensamentos consistem em atitudes proposicionais e que toda atitude proposicional possui dois aspectos distintos: o aspecto de conteúdo e o aspecto propriamente atitudinal. ${ }^{9}$ Dretske concede que cada um de nós tem acesso direto e imediato aos conteúdos de seus pensamentos e crenças, isto é, que ao pensarmos e crermos sabemos imediata e diretamente em que cremos ou em que pensamos, mas recusa-se a aceitar que a consciência desse conteúdo implique a consciência do aspecto atitudinal envolvido nessa atitude proposicional. Assim, de acordo com ele, quando Maria pensa que está chovendo agora, ela tem consciência do conteúdo do seu pensamento - a saber, que está chovendo agora -, mas essa consciência não é suficiente para fundar a consciência de que ela está pensando que está chovendo agora, pois essa "segunda" consciência inclui obviamente elementos que não se encontram presentes na "primeira" consciência. Quando Maria tem

${ }^{9}$ Ver a esse respeito Dretske (1995), Dretske (2003b), Bernecker (1996) e Bernecker (1997). 
consciência de que ela pensa que $P$ essa consciência diz respeito não apenas ao pensamento de que $\mathrm{P}$ - quer dizer, ao conteúdo intencional pensado $\mathrm{P}$-, mas sim também ao fato de que ela, Maria, está pensando em P. A consciência ou o saber de que $\mathrm{P}$ vem a ser o estado interno no qual ela se encontra - estando ela, portanto, pensando o pensamento $\mathrm{P}$ - não consiste em um conteúdo presente no pensamento $\mathrm{P}$, conteúdo este do qual Maria se tornaria consciente ao se tornar consciente do pensamento P.

A consciência reflexiva de segunda ordem - a consciência que um sujeito tem de que ele está pensando um determinado pensamento - não reduplica simplesmente, destarte, de acordo com Dretske, o conteúdo da consciência objetual de primeira ordem, pois ela abarca também uma referência, ausente nesta última, ao sujeito do estado interno e ao fato de que ele entretém certo pensamento. ${ }^{10}$ Este aspecto atitudinal dessa consciência de que se pensa um certo pensamento não faz parte do conteúdo mesmo do pensamento pensado, não sendo legítimo, portanto, considerar, tal como Burge ${ }^{11}$ equivocadamente o faz, que o conteúdo da consciência de primeira ordem é simplesmente assumido pela consciência reflexiva de segunda ordem.

Há de se ressaltar nesse ponto que a estratégia de considerar essa dimensão atitudinal como sendo ela mesma um conteúdo proposicional pensado pelo sujeito e que gozaria, portanto, do privilégio de poder ser acessada por meio de um procedimento introspectivo - vale dizer, direta e imediatamente - está, aos olhos de Dretske, fadada ao fracasso, pois o

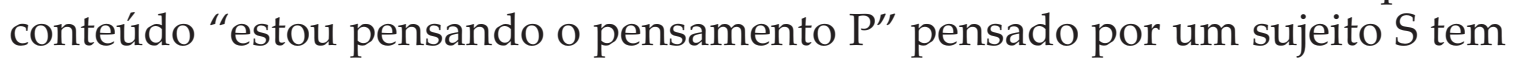
ele mesmo de ser tomado por esse sujeito como sendo um pensamento referido a ele próprio, isto é, como sendo um pensamento efetivamente pensado por esse sujeito, caso contrário esse conteúdo não pode garantir por si só que o sujeito esteja de fato pensando o pensamento "estou pensando o pensamento $\mathrm{P}^{\prime \prime}$. A razão principal apresentada por Dretske para descartar essa possibilidade diz respeito ao fato de os conteúdos desse tipo consistirem em proposições. Uma vez que as proposições podem ser verdadeiras ou falsas, o acesso direto e imediato a um conteúdo proposicional não aumenta por si só a probabilidade de que algo seja um fato. ${ }^{12}$

\footnotetext{
10 "Our question, though, is not a question about content. It is not a question about how we know, in the case of thought, what we are thinking. It is a question about how we know we are thinking it - a question about the attitudinal aspect of thought (see Bernecker (1996), Dretske (1995)). "It is a question about how one gets from what one thinks - that there is beer in the fridge - to a fact about oneself - that one thinks there is beer in the fridge. What you see - beer in the fridge - doesn't tell you that you see it, and what you think - that there is beer in the fridge - doesn't tell you that you think it either.", in Dretske (2003b), p. 2.

${ }^{11}$ Ver a esse respeito Burge (2007a).

12 "When you think there is nothing you are aware of that indicates that you are thinking. What you are aware of that indicates you are thinking. What you are aware of (or acquainted with) is a proposition and propositions do not indicate anything. Unlike facts (conditions, states of affairs), propositions can be either true or false, and a proposition with indeterminate truth-value does not increase the probability that anything else is true." Dretske (2011), p. 431.
} 
Assim o conteúdo proposicional "estou pensando o pensamento $\mathrm{P}$ " não garante por si só o ato de pensar que se está pensando o pensamento $\mathrm{P}$ por parte do sujeito.

Ao tentar incluir o ato de pensar no conteúdo pensado através do recurso a um pensamento de ordem superior que teria como conteúdo o aspecto de conteúdo e o aspecto atitudinal do pensamento de ordem imediatamente inferior incorrer-se-ia inevitavelmente em um regresso ao infinito, pois cada pensamento de um nível superior assumido teria de ser ele mesmo referido ao sujeito, o que exigiria que se pressupusesse um novo pensamento de ordem imediatamente superior que incluísse em seu conteúdo o conteúdo e a atitude desse pensamento, e assim ao infinito.

Apesar de Dretske não tratar explicitamente dela, julgamos que uma outra solução a ser descartada nesse contexto é aquela que considera que pensamentos do tipo "eu penso" são autoverificáveis por consistirem suas negações em contradições performativas. ${ }^{13}$ A ideia central envolvida no recurso a essa noção de contradição performativa para justificar o conhecimento de mim mesmo como sendo um sujeito que pensa pensamentos com determinados conteúdos é a de que eu não posso duvidar que penso sem pensar, dado que a dúvida acerca de me encontrar no estado mental de pensar w pensamento. Assim, não posso afirmar que é dubitável que eu penso, pois a mera realização do ato de pensar esse conteúdo de pensamento como dubitável já o torna verdadeiro, sendo, portanto, ao contrário do que diz Dretske, plenamente fundamentada a afirmação de que sei que penso.

A dificuldade, no que diz respeito ao nosso ponto, é que essa posição toma como dado exatamente o que se encontra problematizado na discussão que ora travamos, a saber, qual seria a natureza dos meios de que dispomos para fundamentarmos a assunção de que nós somos sujeitos de atos mentais, isto é, que pensamos pensamentos com determinados conteúdos. $\mathrm{O}$ apelo ao aspecto performativo dos pensamentos pressupõe justamente que não seja de maneira nenhuma problemático dizer de mim mesmo que sei que me encontro em um determinado estado mental, isto é, dizer de mim mesmo que sei que estou pensando um determinado pensamento. $\mathrm{O}$ pensamento é compreendido aqui, de partida, tanto como um conteúdo acessado pelo sujeito quanto como um ato mental que possibilita ao sujeito conhecer esse conteúdo e, ao mesmo tempo, saber que ele se encontra em um estado mental com esse conteúdo.

O ponto nevrálgico da argumentação de Dretske dirige-se justamente contra a pressuposição de que por meio do apelo à introspecção possamos

\footnotetext{
${ }^{13}$ A reconstrução que Hintikka faz do argumento do cogito cartesiano vai nessa direção. Ver Hintikka (1962).
} 
conhecer fatos - por exemplo, o fato de que pensamos - e não apenas conteúdos. Os pensamentos são, para Dretske, transparentes, possibilitando aos sujeitos que os entretêm a absorção do conteúdo representacional ou informacional por eles veiculados, mas não possibilitam nenhum tipo de conhecimento acerca de fatos relativos a esses sujeitos mesmos. Não há, por assim dizer, um conhecimento introspectivo dos pensamentos estados internos dos sujeitos que acedem aos seus conteúdos. Não se passa, assim, de acordo com Dretske, sem mais do conhecimento imediato e direto das representações ou informações acessadas pelo sujeito ao conhecimento do ato de acesso do sujeito a essas informações. O conhecimento dos conteúdos não traz consigo nem implica um conhecimento dos atos por meio dos quais essas informações e representações tornam-se acessíveis para os sujeitos.

Se sabemos que pensamos e, portanto, se sabemos que não somos zumbis - e Dretske, gostaríamos de sublinhar mais uma vez, não duvida nenhum momento que o saibamos - esse conhecimento não se dá por meio de introspecção, isto é, não se dá através do acesso ao conteúdo representacional de uma proposição, senão que por meio de uma outra via qualquer.

O reconhecimento desse fato pode ser compreendido, cremos, contra Dretske, como uma evidência de uma limitação inerente à sua teoria acerca do conteúdo dos estados mentais, pois se Dretske reconhece um certo fato epistêmico - o fato de que possuímos o conhecimento de que não somos zumbis - do qual sua teoria não consegue dar conta, isso parece indicar que essa concepção apresenta um ponto cego em relação a uma questão crucial para cada um de nós, a saber, o conhecimento que temos de nós mesmos como sujeitos dotados de mentes.

O ceticismo conciliatório de Dretske acerca do conhecimento de si talvez consista, então, em uma posição mandatória unicamente em função da concepção representacional externalista e informacional acerca dos conteúdos mentais assumida por ele. O caráter aparentemente contraintuitivo, e mesmo paradoxal ${ }^{14}$, desse ceticismo quiçá indique a necessidade de apelarmos a uma concepção distinta da de Dretske não acerca da fonte do conhecimento de si, mas sim acerca da natureza mesma desse conhecimento. ${ }^{15}$ Uma possível via para a dissolução desse impasse teórico reside, a nosso ver, na consideração do conhecimento de si como sendo

\footnotetext{
${ }^{14}$ Esse termo é utilizada pelo próprio Dretske para caracterizar a impressão causada pela sua concepção: "If we think, we can know - in a privileged and authoritative way - what we think. What we can't know, at least not in the same authoritative way, is that we are thinking it. If we know it, we know it in some way other than introspection. (...) I know this conclusion sounds paradoxical.", Dretske 2003a, p. 136-7.

${ }^{15}$ Grundmann considera, não sem uma certa dose de sarcasmo, que Dretske fornece involuntariamente em seus textos uma redução ao absurdo do externalismo associado à concepção informacional. Conferir: Grundmann (2012), p. 136.
} 
um saber de natureza não-reflexiva e não-proposicional. Evidentemente, por uma questão de espaço e de foco, não nos dedicaremos neste texto a uma análise crítica dessa concepção do conhecimento de si, mas o faremos em textos futuros.

\section{Bibliografia}

BERNECKER, S. (1997), “Die Grenzen des Selbstwissens", Zeitschrift für philosophische Forschung, 51, 216-230.

BERNECKER, S. (1996), "Externalism and the Attitudinal Component of Self-Knowledge", Nôus, 30, 1996, 262-275.

BERNECKER, S. (2000), "Knowing the World by Knowing One's Mind", Synthese, 123, 2000, 1-34.

BERNECKER, S. (2012), "Wissen zweiter Ordnung und Zombies", in Newen, A. \& Vosgerau, G. (Hrsg.), Den eigenen Geist kennen. Selbstwissen, privilegierter Zugang und Autorität der ersten Person, Mentis, Paderborn, 119-134.

BURGE, T. (2007a), "Authoritative Self-Knowledge and Perceptual Individualism". In Burge, T., Foundations of Mind, Oxford University Press, Oxford, 2007.

BURGE, T. (2007b), "Individualism and the Mental". In Burge, T., Foundations of Mind, Oxford University Press, Oxford, 2007.

DESCARTES, R, Oeuvres de Descartes, Ed. C. Adam and P. Tannery,vols. I- XI, Libraire Philosophique J. Vrin, Paris, 1964-1974.

DESCARTES, R. (1953), Oeuvres et Lettres, Gallimard, Paris.

DESCARTES, R. (1985), The Philosophical Writings of Descartes, Vol. I-III, Cambridge University Press, Cambridge.

DRETSKE, F. (2012a), "Awareness and Authority: Skeptical Doubts about SelfKnowledge", in Smithies, D. \& Stoljar, D., Introspection and Consciousness, Oxford University Press, Oxford, 49-64.

DRETSKE, F. (2012B), “DOUBTS ABOUT COGITO”, GRAZER PHILOSOPHISCHE STUDIEN, 84, 2012, 1-17.

DRETSKE, F. (2003a), "Externalism and Self-Knowledge", in Nuccetelli, S. (ed.), New Essays on Semantic Externalism and Self-Knowledge, MIT Press, Cambridge, MA, 2003, 131-142.

DRETSKE, F. (2003b), “How Do You Know You Are Not a Zombie”, in Gertler, B., Privileged Access and First Person Authority, Ashgate, Burlington, 1-13.

DRETSKE, F. (2004), "Knowing What You Think vs. Knowing That You Think It", in Schantz, R. (ed.), The Externalist Challenge, Walter de Gruyter, Berlin - New York, 390-399. 
DRETSKE, F. (1995), Naturalizing the Mind, MIT Press, Cambridge, MA.

DRETSKE, F. (2011), "Skeptical Doubts about Self-Knowledge", in Bernecker, S. \& Pritchard, D. (ed.), The Routledge Companion to Epistemology, Routledge, London, 425-432.

DRETSKE, F. (2000) “The Mind's Awareness of Itself”, in Dretske, F., Perception, Knowledge and Belief. Selected Essays, Cambridge University Press, Cambridge, 158-177.

GRUNDMANN, T. (2012), "Warum Ich weiss, dass Ich kein Zombie bin”, Newen, A. \& Vosgerau, G. (Hrsg.), Den eigenen Geist kennen. Selbstwissen, privilegierter Zugang und Autorität der ersten Person, Mentis, Paderborn,135-148.

HINTIKKA, J. (1962), “Cogito, ergo sum: Inference or performance?”, Philosophical Review 71, 3-32.

MARQUES, E. (2010), "Conteúdo e Autoridade de Primeira Pessoa". In: Silva Filho. W. (org.). Mente, Linguagem e Mundo, Alameda, São Paulo, 2010, pp. 75-86.

MARQUES, E. \& Muzitano, C. (2014), “Externalismo e Autoconhecimento”, Sképsis, 10, 2014.

Endereço dos Autores:

Av. Epitácio Pessoa, 1834, Apt. 104 - Lagoa

22411-072 Rio de Janeiro - RJ

edgarm@terra.com.br

kachediak@gmail.com 